\title{
Parasitology
}

\section{Seventy-five years of solicitude}

\section{Anne Keymer and Don Bundy}

IN 1913, the Rockefeller Foundation accepted the "urgent invitation" of the British Colonial Office to undertake a worldwide campaign of hookworm control. Six years later, the foundation's researchers had obtained estimates suggesting that almost 1,000 million people, more than half the world's population at that time, were infected. They concluded, with a hint of ambitious philanthropy, that "this one disease may go far to explain the retardation of backwood people" (Ettling, J. The Germ of Laziness, Harvard University Press, 1981). The campaign achieved notable successes (Fig. 1) and laid the foundation of modern helminth epidemiology, but this momentum was not sustained beyond its lifetime. Global estimates of hookworm infection still stand at 1,000 million cases, though these now represent only one quarter of the total world population. The results of research reported at a recent meeting* suggest that the time has come for renewed effort:

Hookworm disease is the term traditionally used to describe infection with either of two species of intestinal nematode, Necator americanus or Ancylostoma duodenale (Fig. 2). But there are important differences in the reproductive strategies of the two species (G. A. Schad, University of Pennsylvania): A. duodenale is more pathogenic than $N$. americanus (as judged by the rate of associated blood loss) and has a higher reproductive rate. Human volunteer studies suggest, as might be predicted, that $N$. americanus has the longer lifespan (E. A. Ottesen, National Institutes of Health). A. duodenale, unlike $N$. americanus, can infect the host orally as well as by skin penetration, and is capable of arrested development in the tissues of the host and perhaps of transmammary transmission from mother to offspring. These and other differences between the two species almost certainly result in divergent epidemiological patterns, the description of which awaits species-specific diagnostic tests for the eggs liberated in the faeces of infected patients, and for larvae in the contaminated environment. The possibility that community control may shift the balance between the two parasites, for example by selecting for arrested larvae resistant to the effects of anthelminthic drugs, has not yet been adequately considered.

The best prospects for a species-specific diagnostic test seem to be the heat-shock proteins that constitute species-specific

* Hookworm Disease: Current Status and New Directions. organized by G.A. Schad and K.S. Warren. Rockefeller Foundation, Bellagio. Italy, 26-30 September 1988. immunodominant antigens in several human disease agents (N. Agabian, University of California, San Francisco). Although there has been some progress, studies of the functional significance of immune responses against human hookworms have been hampered by the absence of epidemiologically defined sera. The surface antigens of $N$. americanus are highly immunogenic, and may shield the underlying collagen which constitutes the main structural component of the parasite's cuticle. Analysis of the sera already available indicates a marked diversity in antigen recognition between patients from endemic areas. If the antibody responses against cuticular

\section{IMAGE UNAVAILABLE FOR COPYRIGHT REASONS}

Fig. 1 A 7-year-old Tamil boy before treatment for hookworm disease (left). His weight was 32 pounds. Right, the same boy 6 months later, after being cured. Photograph taken in 1920 by W. Rose

collagen observed in some patients indicate immunologically mediated parasite damage, the prospects for vaccine development may be better than has previously been supposed (D. I. Pritchard, University of Nottingham).

Hookworms produce secretory antigens which may be of functional significance. Acetylcholinesterase, for example, may modulate the effects of the host's immune responses as well as acting as a biochemical holdfast for the parasites. Proteases which cleave $\operatorname{IgA}$ (one of the putative immunological effectors in the intestine) have also been demonstrated, and infected patients may have reduced levels of intestinal $\mathrm{IgA}$ which return to normal after treatment to remove intestinal worms (Ottesen). Determining the role of these responses in naturally acquired infection depends on further understanding of immunology and epidemiology.

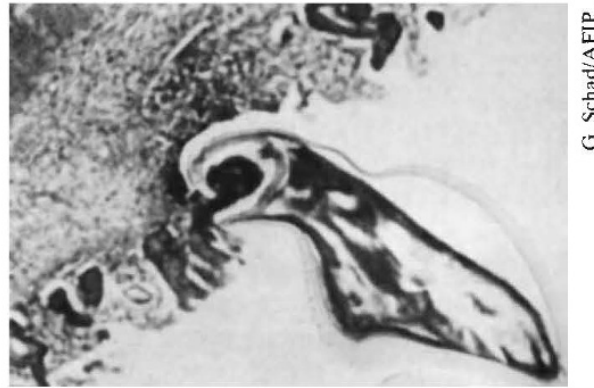

Fig. 2 Section through the lumen showing part of the host intestine in the mouth of $A$. duodenele. The patient died of intestinal blood loss.

Hookworms are known to exert various insidious effects on health, and have been widely associated with the iron-deficiency anaemia which affects 350 million women of child-bearing age. The role of hookworm infection as a contributory factor in the maternal and fetal morbidity associated with anaemia is now beyond dispute (D. W. T. Crompton, University of Glasgow). There is clearly a correlation between hookworm infection and impairments in growth and physical fitness. A study of Kenyan schoolchildren (L. S. Stephenson, Cornell University), for example, demonstrated a significant difference in growth rate between children allocated at random between placebo and anthelminthic treatments. The improvement in weight gain in the treated children (about $1.3 \mathrm{~kg}$ ) is correlated with pretreatment hookworm egg counts, occurred despite continual exposure to reinfection and incomplete cure, and demonstrates one of the benefits that might accrue from effective control

Mortality from acute infectious diseases is gradually coming under control, which means that morbidity from chronic infections such as hookworm assumes greater importance. Control of this morbidity is emerging as a new challenge to publichealth workers in developing countries. The revolution in primary health care and the development of safe and effective broad-spectrum drugs provide new opportunities to meet this challenge in a costeffective way, notably by the integration of the control of hookworm and other chronic nematode infections in existing health-care delivery systems. National schistosomiasis control programmes, for example, provide an existing infrastructure for disease surveillance and drug delivery, and often a sanitation component which may influence the transmission of a wide range of enteric infections. Whatever the combination of interventions chemotherapy, sanitation or even vaccination - current research in epidemiology greatly strengthens the prospects for an integrated strategy.

Anne Keymer is in the Department of Zoology, University of Oxford, Oxford OXI 3PS, Don Bundy is in the Parasite Epidemiology Research Group, Imperial College, London $S W 72 B B, U K$. 